\title{
Inertiale Lage- und Bewegungsverfolgung für instrumentierte Strömungsfolger zur Strömungscharakterisierung
}

\author{
Lukas Buntkiel ${ }^{1}$, Sebastian Reinecke ${ }^{1}$ und Uwe Hampel ${ }^{1,2}$ \\ ${ }^{1}$ Helmholtz-Zentrum Dresden-Rossendorf, HZDR \\ ${ }^{2}$ Professur für Bildgebende Messverfahren für die Energie- und Verfahrenstechnik, TU Dresden \\ Kontakt: I.buntkiel@hzdr.de
}

\section{Einleitung}

Zur Untersuchung der ablaufenden Prozesse in großen Behältern wie z.B. Biogasfermentern, Bioreaktoren und Belebtschlammbecken, wurde am HZDR das Konzept instrumentierter, strömungsfolgender Sensorpartikel entwickelt [1, 2]. Bisher wurden damit die Strömungsverhältnisse mit probabilistischen Auswertemethoden ausschließlich basierend auf der Messung der vertikalen Position als Funktion des hydrostatischen Drucks charakterisiert und $\mathrm{Pa}$ rameter einfacher Prozessmodelle für Anlagen im Labor- und Pilotmaßstab bestimmt.

Derzeit wird das Konzept des Sensorsystems mit dem Ziel weiterentwickelt, eine räumliche Lage- und Bewegungsverfolgung in Bioreaktoren basierend auf Inertialsensorik zu realisieren. Dies umfasst sowohl die dafür zu qualifizierenden Sensoren für $\mathrm{Be}-$ schleunigung, Drehrate, Magnetfeld sowie Umgebungsdruck als auch die Softsensor-Algorithmen zur Bestimmung der Lage und der Bewegung. Damit sollen zukünftig Auftriebsmanöver der Sensorpartikel autonom ablaufen und die räumliche Strömungscharakterisierung in Bioreaktoren erfolgen.

Im Folgenden werden drei Softsensor-Algorithmen zur Bewegungsverfolgung in der $x-y$-Ebene vorgestellt und anhand von Simulation und Messungen miteinander verglichen. Der Fokus liegt dabei auf der Lagebestimmung. Die vertikale Bewegungsverfolgung ist durch den integrierten Drucksensor bereits mit einem absoluten Fehler von maximal ca. $60 \mathrm{~cm}$ messbar und soll daher nicht weiter betrachtet werden.

\section{Softsensoralgorithmen zur Bewegungs- verfolgung}

Die Sensorik des bestehend aus Gyroskop und Beschleunigungssensor erweitert um das Magnetometer und den Drucksensor entspricht dem eines klassischen Trägheitsnavigationssystems. Die Sensoren messen bis auf den Drucksensor in den Koordinaten des Sensorpartikels. Die Position $\boldsymbol{p}$, Geschwindigkeit $\boldsymbol{v}$, Beschleunigung $\boldsymbol{a}$ und Lage $\boldsymbol{q}$, dargestellt als Einheitsquaternion (es gilt $\boldsymbol{R} \triangleq \boldsymbol{R}\{\boldsymbol{q}\}$ ), im Koordinatensystems des Behälters ist über das Zustandsraummodell

$$
\begin{aligned}
\dot{p} & =v \\
\dot{v} & =R\left(a_{m}-a_{b}-a_{n}\right)+g \\
\dot{q}=0,5 * \quad & \otimes\left(\omega_{m}-\omega_{b}-\omega_{n}\right) \#(1) \\
\dot{a}_{b} & =a_{w} \\
\dot{\omega}_{b} & =\omega_{w} \#(2)
\end{aligned}
$$

mit den Messungen $\boldsymbol{a}_{\boldsymbol{m}}$ und $\boldsymbol{\omega}_{\boldsymbol{m}}$ im Sensorkoodinatensystem verbunden. Dabei sind $\boldsymbol{a}_{b}$ und $\boldsymbol{\omega}_{b}$ die Sensorbias und $\boldsymbol{a}_{n}, \boldsymbol{\omega}_{n}, \boldsymbol{a}_{w}$ und $\boldsymbol{\omega}_{w}$ das Sensorrauschen bzw. das Rauschen, dass einen Biasdrift hervorruft. Das Quaternion $\boldsymbol{q}$ ist mit

$$
\boldsymbol{q}=q_{w}+q_{x} i+q_{y} j+q_{z} k=\left[\begin{array}{l}
q_{w} \\
\boldsymbol{q}_{v}
\end{array}\right]=\left[\begin{array}{c}
\cos \theta / 2 \\
\mathrm{e}_{\mathrm{x}} \sin \theta / 2 \\
\mathrm{e}_{\mathrm{y}} \sin \theta / 2 \\
\mathrm{e}_{\mathrm{z}} \sin \theta / 2
\end{array}\right]
$$

parametrisiert, wobei $\theta$ der Drehwinkel und $\boldsymbol{e}$ die normierte Drehachse ist. Aus dem Modell ist ersichtlich, dass die Lage besonders kritisch für eine korrekte Schätzung der Beschleunigung, Geschwindigkeit und Position ist, da mit dieser die Beschleunigung über die Rotationsmatrix $\boldsymbol{R}$ bzw. das Quaternion $\boldsymbol{q}$ ins Behälterkoordinatensystem überführt wird. Aus diesem Grund werden im Folgenden drei Algorithmen zur Lageschätzung vorgestellt, die eine Schätzung des Bias des Gyroskops ermöglichen, da diese im Allgemeinen nur kurzzeitstabil sind. Das sind das Nichtlineare Komplementärfilter (Nonlinear Complementary Filter, NCF), in der Formulierung als Direktes und Passives Komplementärfilter (DCF, PCF) und ein linearisiertes Kalman-Filter (engl. Error State Kalman Filter ESKF).

Das Nichtlineare Komplementärfilter aus [3] ist auf der speziellen Orthogonalen Gruppe (SO(3)), dem Raum der Dreh- oder Rotationsmatrizen, formuliert, sodass durch die Lagefilterung die Bedingungen für Rotationsmatrizen $\left(\operatorname{det}(\boldsymbol{R})=1\right.$ und $\boldsymbol{R}^{T}=\boldsymbol{R}^{-1}$ ) nicht verletzt werden. Das Zustandsraummodell des DCF in Quaternionendarstellung ist

$$
\begin{gathered}
\dot{\boldsymbol{q}}=\frac{1}{2} \widehat{\boldsymbol{q}} \otimes \boldsymbol{p}\left\{\widetilde{\boldsymbol{R}}\left(\boldsymbol{\omega}_{m}-\widehat{\boldsymbol{\omega}}_{b} \dot{)}+2 k_{P} \tilde{q}_{w} \widetilde{\boldsymbol{q}}_{v}\right\} \#(3)\right. \\
\dot{\widehat{\boldsymbol{\omega}}}_{b}=-2 k_{I} \tilde{q}_{w} \widetilde{\boldsymbol{q}}_{v},
\end{gathered}
$$

mit dem Fehlerterm

$$
\widetilde{\boldsymbol{q}}=\widehat{\boldsymbol{q}}^{-1} \otimes \boldsymbol{q}_{m}=\left[\begin{array}{l}
\tilde{q}_{w} \\
\widetilde{\boldsymbol{q}}_{v}
\end{array}\right]
$$

und dem Proportial- und Integralfaktor $k_{P}$ und $k_{I}$. 
Durch Umformulierung in Gl. (3) wird daraus der PCF

$$
\begin{aligned}
& \dot{\boldsymbol{q}}=\frac{1}{1} \widehat{\boldsymbol{q}} \otimes \boldsymbol{p}\left\{\boldsymbol{\omega}_{m}-\widehat{\boldsymbol{\omega}}_{b}+2 k_{P} \tilde{q}_{w} \widetilde{\boldsymbol{q}}_{v}\right\} \\
& \dot{\boldsymbol{\boldsymbol { \omega }}}_{b}=-2 k_{I} \tilde{q}_{w} \widetilde{\boldsymbol{q}}_{v} .
\end{aligned}
$$

Für beide Systeme gelten die Anfangsbedingungen $\widehat{\boldsymbol{\omega}}_{b}(0)=\widehat{\boldsymbol{\omega}}_{b 0}$ und $\widehat{\boldsymbol{q}}(0)=\boldsymbol{q}_{0}$. Die Umformulierung entkoppelt die gemessene Drehrate von der rekonstruierten Lage $\boldsymbol{q}_{m}$ und reduziert die Störanfälligkeit des Filters. Die Filterkorrektur wird über die algebraische Rekonstruktion der Lage $\boldsymbol{q}_{m}$ aus der gemessenen Erdbeschleunigung $\boldsymbol{a}_{\boldsymbol{m}}$ und dem gemessenen Erdmagnetfeld $\boldsymbol{B}_{\boldsymbol{m}}$ durchgeführt. Die Lage lässt sich z.B. durch Minimierung des Kostenfunktionals

$$
L(\boldsymbol{R})=\frac{1}{2} \sum_{i}^{n} a_{i}\left\|\boldsymbol{b}_{i}-\boldsymbol{R} \boldsymbol{r}_{i}\right\|^{2} \#(3)
$$

unter den Bedingungen $\operatorname{det}(\boldsymbol{R})=1$ und $\boldsymbol{R}^{T}=\boldsymbol{R}^{-1}$ bestimmen, wobei $\boldsymbol{r}_{i}$ normierte Referenzvektoren, $\boldsymbol{b}_{i}$ die dazugehörigen Messungen in Körperkoordinaten $\left(\boldsymbol{b}_{i}=\boldsymbol{R} r_{i}\right)$ und $a_{i}$ nicht negative Wichtungsfaktoren sind. Eine Lösung über Davenports q-Methode [6] führt auf die Lage $\boldsymbol{q}_{m}$. Für die Messungen des Beschleunigungssensors wird $\widehat{\boldsymbol{a}}_{\boldsymbol{m}}=\frac{\boldsymbol{a}_{m}}{\left|\boldsymbol{a}_{m}\right|}=-\boldsymbol{R}^{T} \boldsymbol{e}_{3}$ angenommen, d.h. es wird nur die Erdbeschleunigung gemessen. Treten im System weitere Beschleunigungen auf, führt das auf einen Lagefehler. Diese Annahme wird insbesondere in rührerfernen Bereichen großer Bioreaktoren genutzt, welche typischerweise den Großteil des durchströmten Behältervolumens ausmachen. Messungen des Magnetometers werden in der Form $\widehat{\boldsymbol{B}}_{m}=\frac{\boldsymbol{B}_{m}}{\left|\boldsymbol{B}_{m}\right|}$ verarbeitet, wobei angenommen wird, dass das Erdmagnetfeld zeitlich unveränderlich ist.

Das linearisierte Kalman-Filter beruht auf dem Prinzip den wahren Systemzustand $\boldsymbol{x}_{\boldsymbol{t}}$ als eine Kombination aus nominellem Zustand $x$ und dem Fehlerzustand $\boldsymbol{\delta} \boldsymbol{x}$ zu begreifen, d.h. $\boldsymbol{x}_{\boldsymbol{t}}=\boldsymbol{x} \oplus \delta \boldsymbol{x}$. Die Lageschätzung basiert auf den Gleichungen (1) und (2). Die drei Zustandsvektoren sind dann

$$
\begin{aligned}
\boldsymbol{x}_{t} & =\left[\boldsymbol{q}_{t}, \boldsymbol{\omega}_{b t}\right]^{T} \\
\boldsymbol{x} & =\left[\boldsymbol{q}, \boldsymbol{\omega}_{b}\right]^{T} \\
\delta \boldsymbol{x} & =\left[\delta \boldsymbol{\theta}, \delta \boldsymbol{\omega}_{b}\right]^{T},
\end{aligned}
$$

mit dem Quaternionenfehler als $\delta \boldsymbol{q}=[1, \delta \boldsymbol{\theta} / 2]^{T}$. Das nominelle Zustandsraummodell ist dann

$$
\dot{\boldsymbol{q}}=\frac{1}{2} \boldsymbol{q} \otimes\left(\boldsymbol{\omega}_{m}-\boldsymbol{\omega}_{b}\right)
$$

$$
\dot{\boldsymbol{\omega}}_{b}=0 \text {, }
$$

und das Fehlerzustandsraummodell

$$
\begin{aligned}
\delta \dot{\boldsymbol{\theta}} & =-\left[\boldsymbol{\omega}_{m}-\boldsymbol{\omega}_{b}\right]_{\times} \delta \boldsymbol{\theta}-\delta \boldsymbol{\omega}_{b}-\boldsymbol{\omega}_{n} \\
\delta \dot{\boldsymbol{\omega}}_{b} & =\boldsymbol{\omega}_{w} .
\end{aligned}
$$

Abbildung 1: Messmodul mit Arduino Due, BNO055 und SD-Kartenspeicher

Ausgehend von diesen beiden Modellen wurde ein linearisiertes Kalman-Filter nach [5] und [6] implementiert. Als Messgleichungen wurden

und

$$
\boldsymbol{h}_{a}(\boldsymbol{x})=\boldsymbol{R}\{\boldsymbol{q}\} \boldsymbol{e}_{3}
$$

verwendet.

$$
\boldsymbol{h}_{B}(\boldsymbol{x})=\boldsymbol{R}\{\boldsymbol{q}\} \boldsymbol{B}_{0}
$$

Der Vorteil des ESKF gegenüber der NCFs ist, dass eine Schätzung der Kovarianz möglich ist und Sensoren mit verschiedenen Abtastraten genutzt werden können. Die NCF basieren auf der rekonstruierten Lage $\boldsymbol{q}_{m}$, und für diese werden mindestens zwei Richtungsvektoren benötigt. Somit ist der langsamste Sensor das limitierende Element. Der Vorteil der NCFs ist die geringere benötigte Rechenleistung im Vergleich mit dem ESKF.

\section{Aufbau des Messsystems}

Aufgrund der geringen Baugröße des Sensorpartikels kommen MEMS-Sensoren zum Einsatz. Als Messeinheit wird der vollintegrierte BNO055 von Bosch Sensortec eingesetzt. Dieser verfügt über ein Gyroskop, einen Beschleunigungssensor und ein Magnetometer. Zur Charakterisierung und Kalibrierung der Sensoren wurde ein Messmodul aufgebaut, welches die Messeinheit über den 12C-Bus mit einem Arduino Due verbindet. Die Messdaten werden über SPI auf einer SD-Karte gespeichert. Der BNO055 wurde gemäß Tab. 1 parametriert. Das komplette Messmodul ist auf einem Rahmen montiert (siehe Abb. 1). Dieses ermöglicht eine vereinfachte Handhabung, Reproduzierbarkeit und Vergleichbarkeit bei der Aufzeichnung von Referenztrajektorien im Labor. Zukünftig soll das Messmodul auch zur Untersuchung der Achsabweichungen, der Nichtlinearität und des Temperaturverhaltens der Inertialsensoren an entsprechenden Versuchsständen appliziert werden. 
Tabelle 1: Einstellungen des BNO055

\begin{tabular}{|l|l|l|l|l|}
\hline Sensor & $\begin{array}{l}\text { Band- } \\
\text { breite }\end{array}$ & $\begin{array}{l}\text { Messbe- } \\
\text { reich }\end{array}$ & $\begin{array}{l}\text { Abtast } \\
\text { tast- } \\
\text { zeit }\end{array}$ & $\begin{array}{l}\text { Auflö- } \\
\text { sung }\end{array}$ \\
\hline Gyroskop & $230 \mathrm{~Hz}$ & $500^{\circ} / \mathrm{s}$ & $0,01 \mathrm{~s}$ & $\begin{array}{l}1 / 64 \\
\% / \mathrm{s} / \mathrm{LSB}\end{array}$ \\
\hline $\begin{array}{l}\text { Accele- } \\
\text { rometer }\end{array}$ & $250 \mathrm{~Hz}$ & $4 \mathrm{~g}$ & $0,01 \mathrm{~s}$ & $\begin{array}{l}0,01 \\
\mathrm{~m} / \mathrm{s}^{\wedge} 2 / \\
\mathrm{LSB}\end{array}$ \\
\hline $\begin{array}{l}\text { Magne- } \\
\text { tometer }\end{array}$ & $25 \mathrm{~Hz}$ & $\mu \mathrm{T}$ & $0,025 \mathrm{~s}$ & $\begin{array}{l}1 / 16 \\
\mu \mathrm{T} / \mathrm{LSB}\end{array}$ \\
\hline
\end{tabular}

MEMS-Sensoren unterliegen einem starken Rauschen im Vergleich zu z.B. optischen Inertialsensoren. Für inertiale Messeinheiten werden im Allgemeinen fünf verschiedene Rauschterme unterschieden [7]: Quantisierungsrauschen, Angle (Velocity) Random Walk, Bias Instability, Rate Random Walk und Rate Ramp. Um die Terme zu identifizieren, hat sich die Allanvarianzanalyse im Zeitbereich als Methode etabliert. Diese ist im Gegensatz zu einer Analyse der spektralen Leistungsdichte einfacher durchzuführen.

Zur Bestimmung der Rauschparameter mittels Allanvarianzanalyse wurde die Methode nach [7] implementiert und eine statische Messung über 7 Stunden durchgeführt. Die Allanstandardabweichung für die Gyroskop- und Beschleunigungssensortriade findet sich in Abb. 2 und Abb. 3. Aus diesen können die einzelnen Rauschterme ermittelt werden. Die auf drei Stellen gerundeten Ergebnisse sind in den Tabellen 2 und 3 dargestellt. Das Quan-

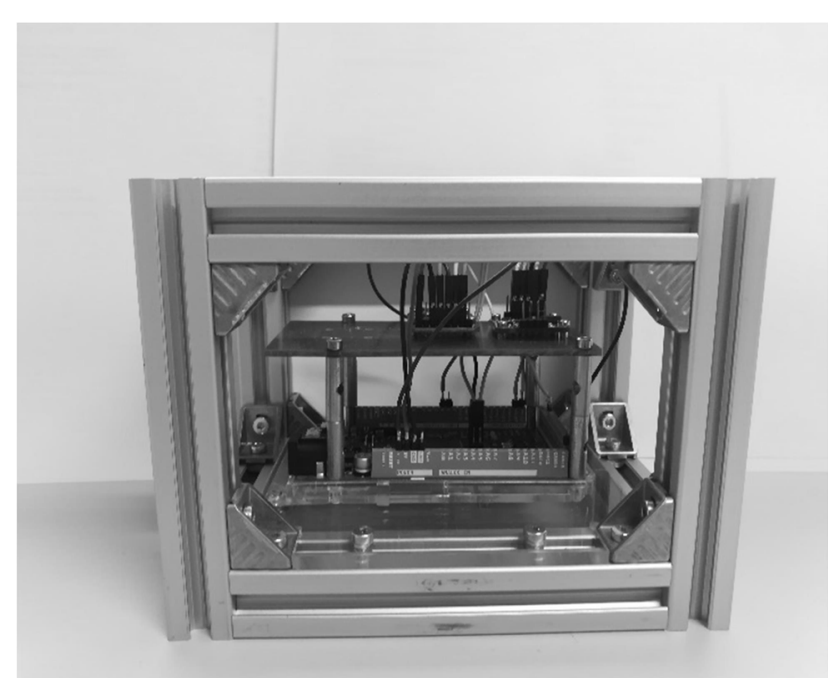

tiserungsrauschen ist das dominierende Rauschen, Angle bzw. Velocity Random Walk sind eine Größenordnung kleiner. Die anderen Terme werden in Abbildung 2: Ermittelte Allanstandardabweichung der Gyroskoptriade des BNO055 den Algorithmen vernachlässigt.

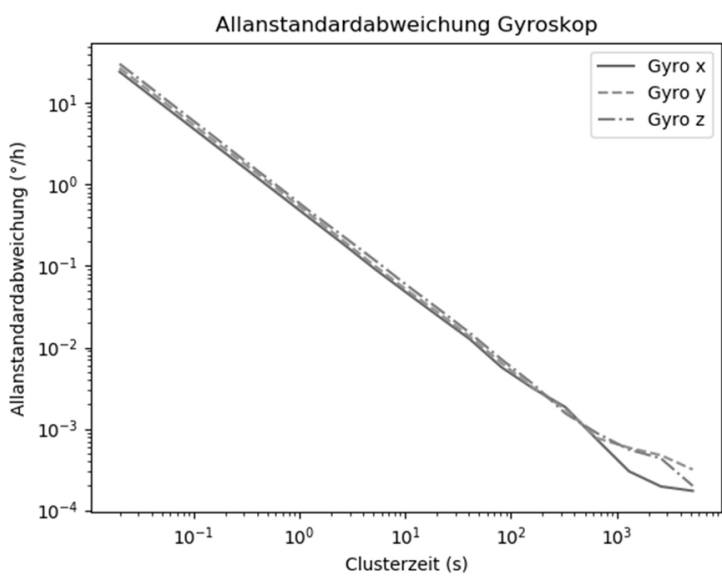

Tabelle 2: Rauschparameter der Gyrokopeinheit

\begin{tabular}{|l|c|c|c|c|}
\hline & $\begin{array}{c}\text { Quantization } \\
\text { Noise }\end{array}$ & $\begin{array}{c}\text { Angle } \\
\text { Random } \\
\text { Walk }\end{array}$ & $\begin{array}{c}\text { Rate } \\
\text { Random } \\
\text { Walk }\end{array}$ & $\begin{array}{c}\text { Rate } \\
\text { Ramp }\end{array}$ \\
\hline $\begin{array}{l}\text { Gyro } \\
\text { x }\end{array}$ & 0,280 & 0,025 & 0,001 & 0,001 \\
\hline $\begin{array}{l}\text { Gyro } \\
\text { y }\end{array}$ & 0,315 & 0,046 & 0,004 & 0,005 \\
\hline $\begin{array}{l}\text { Gyro } \\
\text { z }\end{array}$ & 0,350 & 0,007 & 0,005 & 0,006 \\
\hline
\end{tabular}

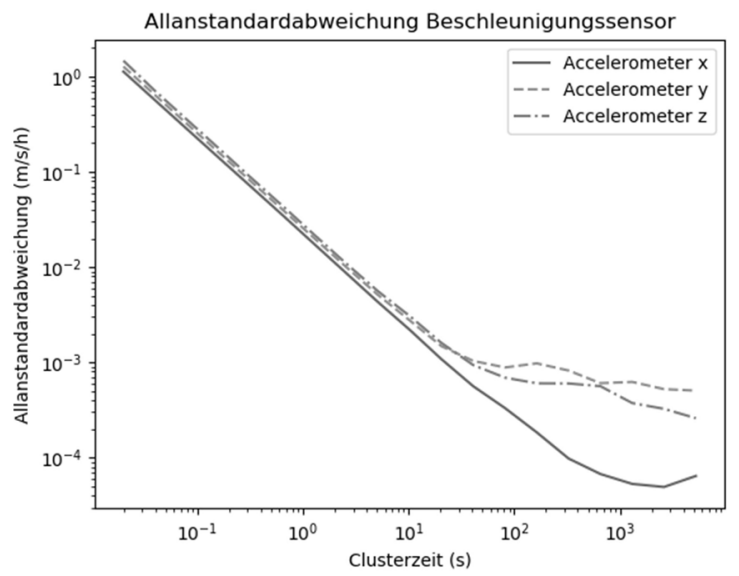

Abbildung 3: Ermittelte Allanstandardabweichung der Beschleunigungssensortriade des BNO055

Tabelle 3: Rauschparameter der Beschleunigungssensoreinheit

\begin{tabular}{|l|c|c|c|c|}
\hline & $\begin{array}{c}\text { Quantizati- } \\
\text { on } \\
\text { Noise }\end{array}$ & $\begin{array}{c}\text { Accelerati- } \\
\text { on Random } \\
\text { Walk }\end{array}$ & $\begin{array}{c}\text { Rate } \\
\text { Rand- } \\
\text { om } \\
\text { Walk }\end{array}$ & $\begin{array}{c}\text { Rate } \\
\text { Ram } \\
\mathrm{p}\end{array}$ \\
\hline $\begin{array}{l}\text { Ac } \\
\text { c } x\end{array}$ & 0,013 & 0,002 & 0 & 0 \\
\hline Ac & 0,015 & 0,007 & 0 & 0 \\
\hline
\end{tabular}




\begin{tabular}{|l|l|l|l|l|}
\hline c y & & & & \\
\hline $\begin{array}{l}\text { Ac } \\
\text { c z }\end{array}$ & 0,016 & 0,004 & 0 & 0 \\
\hline
\end{tabular}

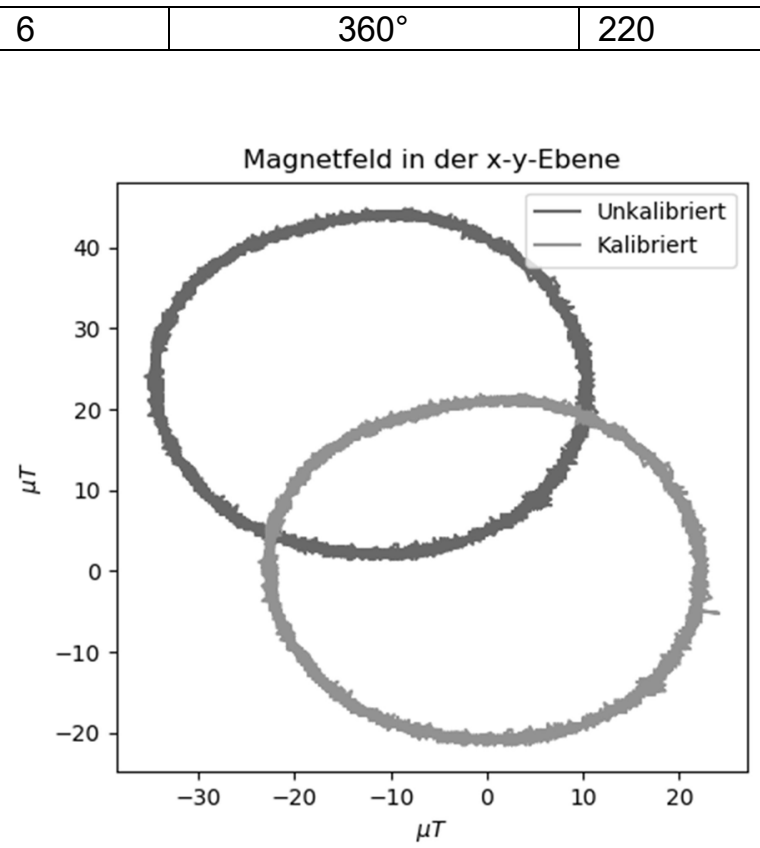

\section{Vergleich der Algorithmen}

Die vorgestellten Algorithmen sollen im Kontext von runden Behältern, in denen überwiegend kreisförmige Bewegungen vorkommen, analysiert werden. Der Fokus liegt dabei auf der x-y-Ebene und dem Lagefehler. Dazu wurden gleichförmige Kreisbewegungen mit verschiedenen Winkelgeschwindigkeiten simuliert und mit dem identifizierten Rauschen überlagert. Die Bewegung startet nach einer Wartezeit von 100 Sekunden. Das Erdmagnetfeld wird mit $\boldsymbol{B}_{0}=[5 ; 8 ; 18,5]$ simuliert und die Anfangsbedingungen sind $\boldsymbol{q}_{0}=[1 ; 0 ; 0 ; 0]$ und $\widehat{\boldsymbol{\omega}}_{b 0}$ ist der Mittelwert über die ersten 60 Sekunden Messzeit, in der keine Bewegung stattfindet. Als Fehlermaß für die Lage- und Biasschätzung wird die Wurzel aus der mittleren quadratischen Abweichung (Root Mean Square Error)

$$
R M S E=\sqrt{\frac{1}{T} \sum_{k=1}^{T}\left(x_{k}-\hat{x}_{k}\right)^{2}}
$$

gewählt. Die Wichtungsfaktoren des Kostenfunktionals Gl.(3) sind die größte Allanvarianz des Beschleunigungssensor aus Tab. 4 und die größte Varianz des Magnetometers geschätzt aus den ersten 60 Sekunden Messzeit. Die Parameter der durchgeführten Simulationen finden sich in Tab. 5. Der Proportional- und Integralanteil des PCF und DCF wurde zu $k_{P}=3,6$ und $k_{I}=0.2$ gewählt

Für die Versuche wurde das System auf einem Drehtisch montiert (siehe Abb. 5) und die Versuche nach Tab. 4 durchgeführt. In jedem Versuch wurden für 10 Minuten Messdaten aufgezeichnet. Die Drehung wurde nach ca. 100 Sekunden gestartet und die kreisförmige Bewegung dauerte jeweils ca. 220 Sekunden. Das Magnetometer unterliegt ebenfalls einem Bias. Dieser führt dazu, dass eine kreisförmige Bewegung nicht um den Ursprung verläuft, sondern einen verschobenen Mittelpunkt hat. Deshalb wurden die aufgenommenen Daten in der x-y-Ebene aufgetragen und der Mittelpunkt bestimmt. Danach wurden alle Daten um den Bias bereinigt (vgl. Abb. 4).

Tabelle 4: Durchgeführte Simulationen und Versuche

\begin{tabular}{|l|c|l|}
\hline $\begin{array}{l}\text { Ver- } \\
\text { such }\end{array}$ & $\begin{array}{c}\text { Winkelgeschwindig- } \\
\text { keit }\end{array}$ & $\begin{array}{l}\text { Umdrehun- } \\
\text { gen }\end{array}$ \\
\hline 1 & $18^{\circ}$ & 13 \\
\hline 2 & $36^{\circ}$ & 25 \\
\hline 3 & $90^{\circ}$ & 65 \\
\hline 4 & $180^{\circ}$ & 120 \\
\hline 5 & $270^{\circ}$ & 180 \\
\hline
\end{tabular}

Abbildung 4: Magnetfeld in der x-y-Ebene

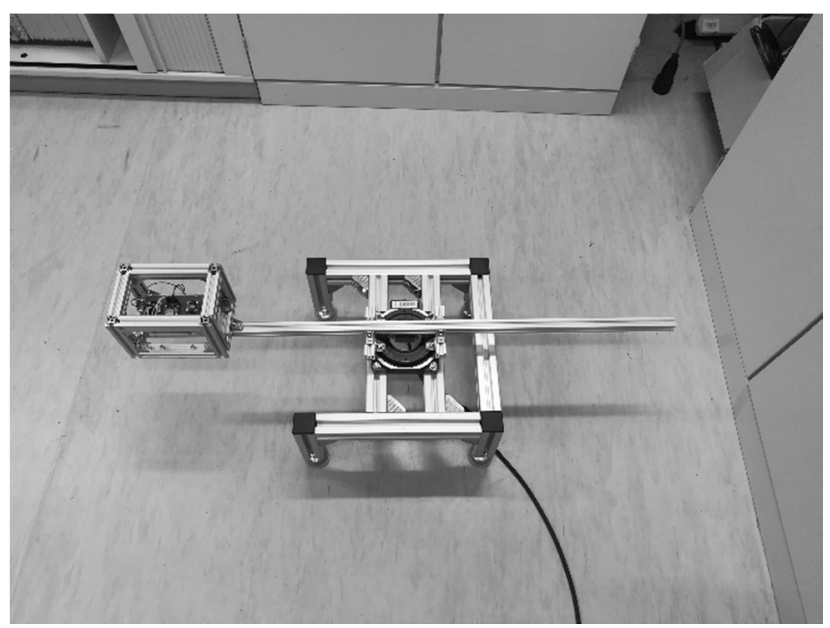

Abbildung 5: Auf Drehtisch montiertes Messmodul

\section{Simulationsergebnisse}

Die Ergebnisse der Simulation finden sich in Tab. 6. Entscheidend für die Anwendung im Sensorpartikel ist eine möglichst genaue Lageschätzung. Aus Tab. 5 ist zu sehen, dass die Lage für alle sechs Versuche geschätzt wird. Zwar steigt die Abweichung mit steigender Winkelgeschwindigkeit an, dies ist allerdings zu erwarten, da die Annahme $\widehat{\boldsymbol{a}}_{\boldsymbol{m}}=$ $\boldsymbol{a}_{m} /\left|\boldsymbol{a}_{m}\right|=-\boldsymbol{R}^{T} \boldsymbol{e}_{3}$ verletzt wird. Der ESKF weist von allen Algorithmen die geringste Abweichung in den Komponenten der Quaternionen auf und steigt vergleichsweise langsam an. Der PCF schätzt die Lage in den ersten drei Versuchen genauso gut wie der DCF. Danach macht der DCF größere Fehler und wird unbrauchbar. Exemplarisch ist die Lageschätzung für die erste Simulation in Abb. 6 dargestellt. 
Der Wankwinkel schwankt zwischen den absoluten Werten $180^{\circ}$ und $-180^{\circ}$, was derselben Lage entspricht, und ist deshalb weggelassen. Der ESKF schätzt eine stabile Lage und kehrt nach dem Ende der Bewegung wieder in die Ausgangslage zurück. Das gilt für alle Versuche und ist für den Anwendungsfall in Bioreaktoren besonders relevant, da am Rührwerk schnelle Bewegungen auftreten, während in über $90 \%$ des Behältervolumens eine sehr langsame Bewegung vorherrscht. Der PCF und DCF hingegen kehren nicht zur Ausgangslage zurück. Dies kann ggf. durch eine Optimierung der Parameter $k_{P}$ und $k_{I}$ erreicht werden. Wie in Abb. 7 dargestellt, ermöglicht es der ESKF, die Beschleunigung in Behälterkoordinaten zu überführen. Die Amplitude ist etwas zu gering, dies lässt sich auf die begrenzte Auflösung des Beschleunigungssensors zurückführen.
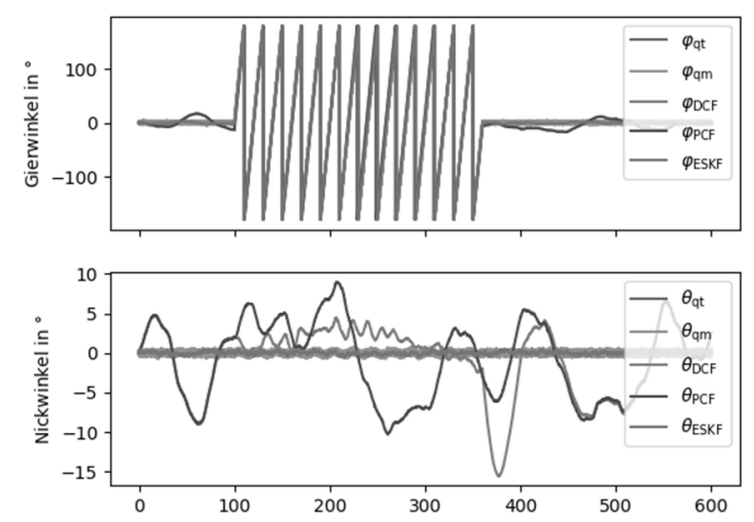

Abbildung 6: Nick und Gierwinkel für die erste Simulation, mit den Indizes $m$ für die rekonstruierte Lage und t für die wahre Lage

Tabelle 5: RMSE von 6 Simulationen für alle Achsen und Algorithmen

\begin{tabular}{|l|l|l|l|l|l|l|l|l|l|l|l|l|}
\hline Versuch & \multicolumn{3}{|c|}{$\mathbf{1}$} & \multicolumn{3}{|c|}{$\mathbf{2}$} & \multicolumn{3}{|c|}{$\mathbf{3}$} & \multicolumn{3}{c|}{$\mathbf{4}$} \\
\hline Algorithmus & ESKF & PCF & DCF & ESKF & PCF & DCF & ESKF & PCF & DCF & ESKF & PCF & DCF \\
\hline RMSE $\left(\boldsymbol{q}_{\boldsymbol{w}}\right)$ & 0,04 & 0.55 & 0.55 & 0.05 & 0.38 & 0.38 & 0.04 & 0,18 & 0,17 & 0,08 & 0,22 & 9,12 \\
\hline $\operatorname{RMSE}\left(\boldsymbol{q}_{\boldsymbol{x}}\right)$ & 0,01 & 0,17 & 0,22 & 0,02 & 0,38 & 0,38 & 0,11 & 0,14 & 0,29 & 0,39 & 0,5 & 0,98 \\
\hline $\operatorname{RMSE}\left(\boldsymbol{q}_{\boldsymbol{y}}\right)$ & 0,02 & 0,17 & 0,22 & 0,03 & 0,37 & 0,25 & 0,1 & 0,46 & 0,37 & 0,36 & 0,61 & 2,15 \\
\hline $\operatorname{RMSE}\left(\boldsymbol{q}_{\boldsymbol{z}}\right)$ & 0,08 & 0,8 & 0,8 & 0,07 & 0,41 & 0,44 & 0,08 & 0,69 & 0,7 & 0,09 & 0,64 & 8,78 \\
\hline $\operatorname{RMSE}\left(\boldsymbol{\omega}_{\boldsymbol{b} \boldsymbol{x}}\right)$ & 2,86 & 5,04 & 2,83 & 2,86 & 8,58 & 2,83 & 5,16 & 3,14 & 2,85 & 9,74 & 4,87 & 7,29 \\
\hline $\operatorname{RMSE}\left(\boldsymbol{\omega}_{\boldsymbol{b} \boldsymbol{y}}\right)$ & 3,43 & 8,23 & 5,45 & 4,01 & 11,96 & 5,74 & 7,45 & 7,79 & 5,13 & 13,75 & 6,94 & 21,36 \\
\hline $\operatorname{RMSE}\left(\boldsymbol{\omega}_{\boldsymbol{b z}}\right)$ & 6,3 & 9,65 & 9,69 & 5,73 & 8,81 & 8,34 & 5,73 & 8,29 & 8,71 & 5,72 & 8,76 & 62,41 \\
\hline
\end{tabular}

Tabelle 5: Fortsetzung

\begin{tabular}{|l|l|l|l|l|l|l|}
\hline Versuch & \multicolumn{3}{|c|}{$\mathbf{5}$} & \multicolumn{3}{c|}{$\mathbf{6}$} \\
\hline Algorithmus & ESKF & PCF & DCF & ESKF & PCF & DCF \\
\hline $\operatorname{RMSE}\left(\boldsymbol{q}_{\boldsymbol{w}}\right)$ & 0,13 & 1,2 & 14,48 & 0,18 & 1,41 & 14,59 \\
\hline $\operatorname{RMSE}\left(\boldsymbol{q}_{\boldsymbol{x}}\right)$ & 0,76 & 0,95 & 1,91 & 1,06 & 1,12 & 2,95 \\
\hline $\operatorname{RMSE}\left(\boldsymbol{q}_{\boldsymbol{y}}\right)$ & 0,69 & 1,51 & 1,86 & 0,92 & 1,67 & 1,84 \\
\hline $\operatorname{RMSE}\left(\boldsymbol{q}_{\boldsymbol{z}}\right)$ & 0,1 & 1,31 & 5,97 & 0,13 & 1,46 & 5,48 \\
\hline $\operatorname{RMSE}\left(\boldsymbol{\omega}_{\boldsymbol{b} \boldsymbol{x}}\right)$ & 29,22 & 8,5 & 12,06 & 67,07 & 4,06 & 10 \\
\hline $\operatorname{RMSE}\left(\boldsymbol{\omega}_{\boldsymbol{b} \boldsymbol{y}}\right)$ & 21,2 & 28,94 & 8,61 & 36,67 & 26,24 & 17,46 \\
\hline $\operatorname{RMSE}\left(\boldsymbol{\omega}_{\boldsymbol{b} \boldsymbol{z}}\right)$ & 6,87 & 10,2 & 12,7, & 6,87 & 10,05 & 11,51 \\
\hline
\end{tabular}

Die Biasschätzung für die erste Simulation ist in Abb. 8 dargestellt. Alle Algorithmen sind in der Lage dem zeitveränderlichen Bias zu folgen. Mit steigenden Winkelgeschwindigkeiten hingegen wird die Schätzung völlig unbrauchbar. Dies liegt an der steigenden Abweichung des Beschleunigungsvektors von der Erdbeschleunigung. Besonders der ESKF fällt negativ auf. Die Schätzung lässt sich verbessern, indem im Filter die Parameter für das Messrauschen des Beschleunigungssensors an die im System auftretenden Beschleunigungen angepasst werden.

\section{Messergebnisse}

Aus den durchgeführten Messungen sind in den Abbildungen 9 und 10 exemplarisch der Gier- und Nickwinkel und die Gyropbiasschätzung auf der zAchse für den 1.Versuch dargestellt. Die Lageschätzung wird von allen Algorithmen zuverlässig durchgeführt. Allerdings zeigt sich, dass die NCFs bei Rückkehr in die Ruhelage noch etwas nachschwingen, während der ESKF sofort die korrekte Lage schätzt. Die Biasschätzung wird ungenau, sobald die Bewegung einsetzt. Hier könnte ein Störgrößenbeobachter Abhilfe schaffen. Der ESKF schwingt aus bisher nicht geklärten Gründen besonders stark. 
Ein Grund könnte in den unkalibrierten Gyroskopund Beschleunigungsdaten liegen. Der Beschleunigungssensor wurde lediglich vom Bias bereinigt.

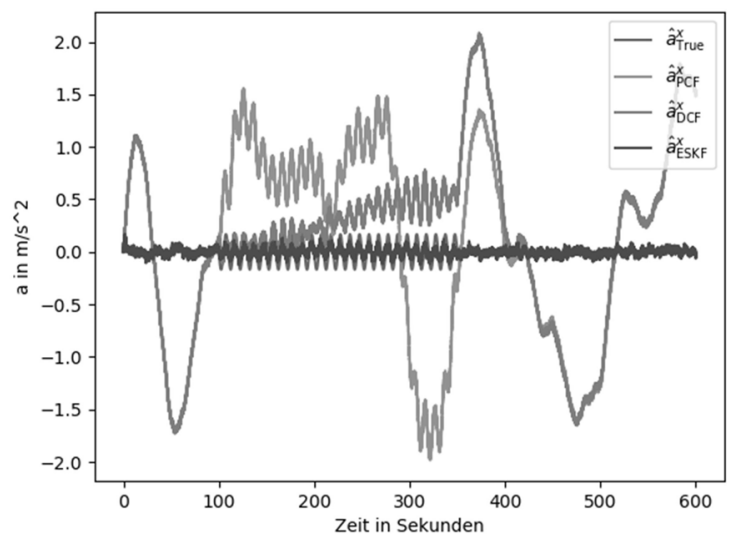

Abbildung 7: Beschleunigung in Behälterkoordinaten auf der $\mathrm{x}$-Achse für die zweite Simulation

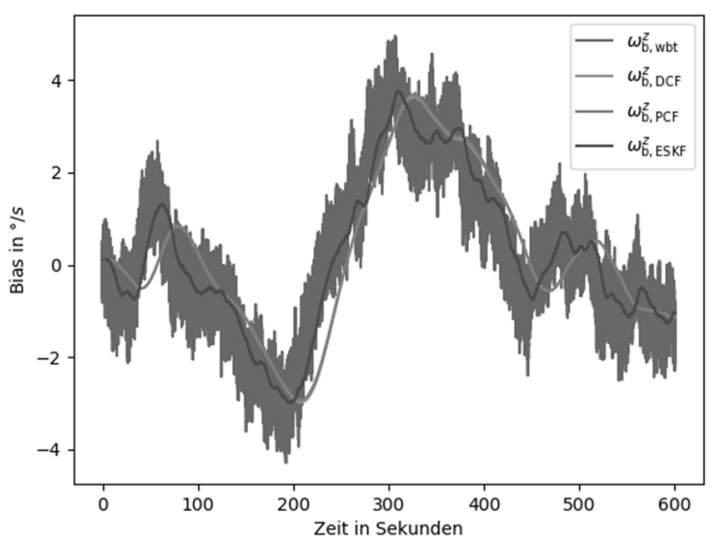

Abbildung 8: Biasschätzung auf der z-Achse für die erste Simulation
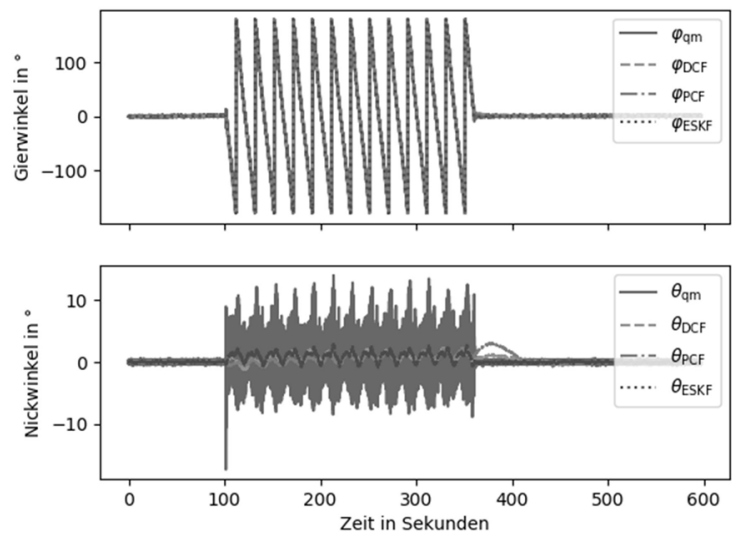

Abbildung 9: Nick und Gierwinkel für die erste Messung

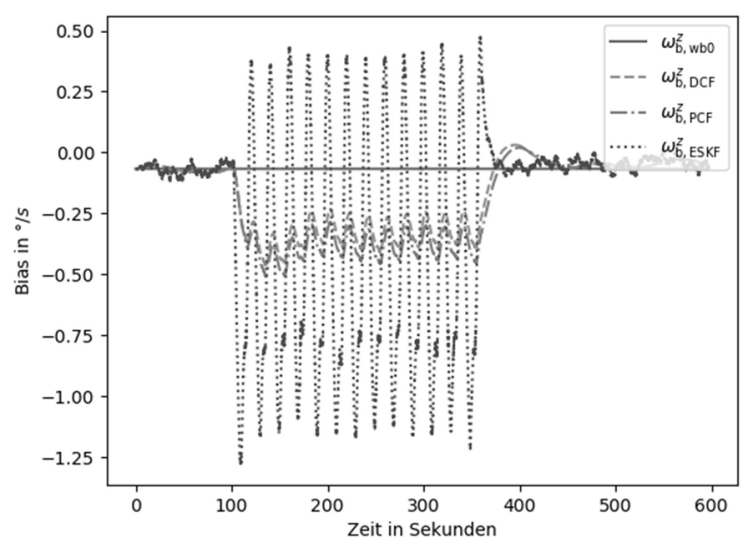

Abbildung 10: Biasschätzung auf der z-Achse für die erste Messung

\section{Zusammenfassung und Ausblick}

Die vorgestellten Algorithmen wurden hinsichtlich ihrer Einsetzbarkeit bei der Lageschätzung in der x$\mathrm{y}$-Ebene untersucht. Insbesondere bei langsamen Bewegungen kann mit allen Algorithmen die Lage geschätzt werden. Der ESKF ist dabei genauer als die NCFs. In den Simulationen wird der Gyroskopbias mit Hilfe des ESKFs zuverlässig geschätzt, für Messungen jedoch nicht. Dieses Verhalten muss weiter untersucht werden. Ebenso wird eine adaptive Anpassung der Beschleunigungssensorvarianz an die jeweils gemessene Beschleunigung vorgeschlagen. Das sollte zu einer besseren Biasschätzung führen. Außerdem wird bei zukünftigen Messungen die IMU vollständig kalibriert werden und es werden verschiedene IMUs erprobt. Insbesondere wird ein Beschleunigungssensor mit einer hinreichenden Auflösung genutzt. Der ESKF wird um eine Geschwindigkeits- und Positionsschätzung, sowie um den Drucksensor erweitert werden.

Die Ergebnisse zeigen, dass die NCDs für diesen Einsatzbereich keine gute Wahl sind, obwohl sie weniger Rechenleistung benötigen.

\section{Danksagung}

Gefördert durch: Bundesministerium für Ernährung und Landwirtschaft auf Grund eines Beschlusses des Deutschen Bundestages (FKZ 22032618)

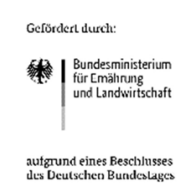

\section{Literatur}

[1] Thiele, S., Da Silva, M. J., Hampel, U.: Autonomous sensor particle for parameter tracking in large vessels, Meas. Sci. Technol., vol. 21(8), 2010 
[2] Reinecke, S. F., Hampel, U.: Instrumented flow following sensor particles with magnetic position detection and buoyancy control, J. Sens. Sens. Syst., vol. 5, 2016, S. 213-22

[3] Mahony, R., Hamel, T., Pflimlin, J.-M.: Nonlinear Complementary Filters on the Special Orthogonal Group,

IEEE Transactions on Automatic Control, Institute of Electrical and Electronics Engineers (IEEE), 2008, 53, 1203-1218

[4] Markley, F. Landis, and Daniele Mortari. Quaternion attitude estimation using vector observations, Journal of the Astronautical Sciences 48.2 (2000): 359-380.

[5] Youn, W., Gadsden, S. A.: Combined QuaternionBased Error State Kalman Filtering and Smooth Variable Structure Filtering for Robust Attitude Estimation IEEE Access, Institute of Electrical and Electronics Engineers (IEEE), 2019, 7, 148989-149004

[6] Sola, J.: Quaternion kinematics for the error-state Kalman filter. arXiv preprint arXiv:1711.02508, 2017

[7] N. El-Sheimy, H. Hou, and X. Niu, Analysis and Modeling of Inertial Sensors Using Allan Variance, IEEE Transactions on Instrumentation and Measurement, vol. 57, no. 1, pp. 140-149, Jan. 2008 